\title{
196.
}

\section{NOTE SUR UN PROBLÈME D'ANALYSE INDÉTERMINÉE.}

[From the Nouvelles Annales de Mathématiques (Terquem and Gerono), :t. Xvi. (1857), pp. 161-165.]

Euler a donné dans le Mémoire: Regula facilis problemata Diophantea per numeros integros expedite solvendi (Comment. Arith. Coll., t. II., p. 263) la solution que voici de l'équation indéterminée

$$
\alpha x^{2}+\beta x+\gamma=\zeta y^{2}+\eta y+\theta
$$

en supposant que l'on ait la solution $x=a, y=b$ de manière que

et en posant

$$
\alpha a^{2}+\beta a+\gamma=\zeta b^{2}+\eta b+\theta,
$$

$$
s=\sqrt{a r^{2}+1}
$$

où $r$ est une quantité quelconque, l'équation sera satisfaite par les valeurs

$$
\begin{aligned}
& x=s a+\zeta r b+\frac{(s-1) \beta}{2 \alpha}+\frac{1}{2} r \eta, \\
& y=\alpha r a+s b+\frac{(s-1) \eta}{2 \zeta}+\frac{1}{2} r \beta ;
\end{aligned}
$$

en effet, on voit sans peine que ces valeurs donnent identiquement

$$
\alpha x^{2}+\beta x+\gamma-\left(\zeta y^{2}+\eta y+\theta\right)=\alpha a^{2}+\beta a+\gamma-\left(\zeta b^{2}+\eta b+\theta\right)=0 .
$$

En supposant de plus que les coefficients $\alpha, \beta, \gamma, \zeta, \eta, \theta$ soient des nombres entiers tels que $\alpha \zeta$ soit un entier positif non carré, on peut toujours déterminer le nombre entier $r$ de manière que $s$ soit un nombre entier; cela étant, et en supposant que $a, b$ soient des entiers, il est évident que $x, y$ seront des nombres rationnels. Euler a de plus remarqué que l'on peut toujours faire en sorte que $x, y$ soient des nombres entiers. En effet, si les formules donnent $x=a^{\prime}, y=b^{\prime}$ des valeurs non entières, en 
substituant dans les formules au lieu de $a, b$ les valeurs $a^{\prime}, b^{\prime}$, on obtiendra pour $x, y$ des valeurs entières; cela se vérifie sans peine.

L'équation indéterminée (2) rentre dans celle-ci

$$
\left(a, b, c, f, g, h \gamma\left(x^{\prime}, y^{\prime}, z^{\prime}\right)^{2}=\left(a, b, c, f, g, h \gamma(x, y, z)^{2}\right. \text {; }\right.
$$

en supposant que la forme ternaire

$$
(a, b, c, f, g, h \gamma x, y, z)^{2}
$$

se transforme en elle-même au moyen d'une substitution linéaire quelconque, on peut supposer que cette substitution soit telle que l'on ait $z^{\prime}=z$; cela étant, en écrivant $z^{\prime}=z=1$ et en mettant de plus $h=0$, l'équation (3) se réduit évidemment à une forme telle que l'équation (2). Or on peut trouver par la méthode générale de M. Hermite la solution convenable de l'équation (3). En supposant, comme à l'ordinaire,

$$
\begin{aligned}
& \mathfrak{A}=b c-f^{2}, \ldots \quad \mathfrak{F}=g h-a f, . . \\
& K=a b c-a f^{2}-b g^{2}-c h^{2}+2 f g h,
\end{aligned}
$$

il faut pour cela écrire

$$
x^{\prime}=2 \xi-x, y^{\prime}=2 \eta-y, z^{\prime}=2 \zeta-z,
$$

et

$$
\begin{aligned}
& a x+h y+g z=a \xi+h \eta+g \zeta \quad-q \llbracket \eta+q \Re \zeta, \\
& h x+b x+f z=h \xi+b \eta+f \zeta+q \xi \xi \quad-q \varpi \zeta,
\end{aligned}
$$

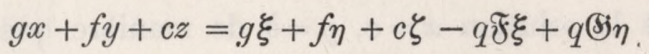

où $q$ est une quantité arbitraire. En effet, en multipliant ces équations par $\xi, \eta, \zeta$ et en ajoutant, on obtient

$$
(a, b, c, f, g, h \gamma \xi, \eta, \xi \chi x, y, z)=(a, b, c, f, g, h \gamma \xi, \eta, \zeta)^{2}
$$

et, au moyen de cette équation et des valeurs

$$
x^{\prime}=2 \xi-x, y^{\prime}=2 \eta-y, z^{\prime}=2 \zeta-z,
$$

on forme tout de suite l'équation (3). De plus, en multipliant les trois équations par $\left(\mathfrak{6}, \mathfrak{F}\right.$, (5) et en ajoutant, on obtient $K z=K \zeta$, c'est-à-dire $z=\zeta$ et de là $z^{\prime}=z$.

Cela étant, les deux équations donnent, en remplaçant $\zeta$ par $z$,

$$
\begin{aligned}
a \xi+(h-q(\mathfrak{5}) \eta & =a x+h y+(g-q \mathfrak{\Im}) z, \\
(h+q(\mathfrak{S}) \xi \quad+b \eta & =h x+b y+(f+q(\mathfrak{S}) z,
\end{aligned}
$$

et de là, en remarquant que

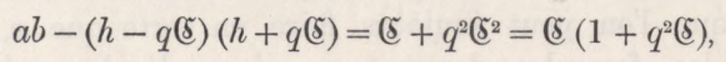


on obtient très-facilement, éliminant successivement $\eta$ et $\xi$,

$$
\begin{aligned}
& \left(1+q^{2}(5) \xi=(1+q h) x+\quad q b y+\left(q f+q^{2}(S) z,\right.\right. \\
& \left(1+q^{2}(5) \eta=-\quad q a x+(1-q h) y+\left(-q g+q^{2} \widetilde{\mho}\right) z\right.
\end{aligned}
$$

et ensuite

$$
\begin{array}{llr}
\left(1+q^{2}(\mathfrak{S}) x^{\prime}=\left(1+2 q h-q^{2}(\mathfrak{S}) x+\right.\right. & 2 q b y+2\left(q f+q^{2}(\mathfrak{S}) z,\right. \\
\left(1+q^{2}(\mathfrak{S}) y^{\prime}=\right. & -2 q a x+\left(1-2 q h-q^{2}(\mathfrak{S}) y+2\left(-q g+q^{2} \mathfrak{\mho}\right) z,\right.
\end{array}
$$

c'est-à-dire, en écrivant $z=z^{\prime}=1$, les valeurs

$$
\left.\begin{array}{llr}
\left(1+q^{2}(5) x^{\prime}=\left(1+2 q h-q^{2}(5) x+\right.\right. & 2 q b y+2\left(q f+q^{2}(5)\right) \\
\left(1+q^{2}(5) y^{\prime}=\right. & -2 q a x+\left(1-2 q h-q^{2}(5) y+2\left(-q g+q^{2} \mathfrak{\zeta}\right)\right.
\end{array}\right\}
$$

satisfont identiquement à l'équation

$$
\left(a, b, c, f, g, h_{\chi} x^{\prime}, y^{\prime}, 1\right)^{2}=\left(a, b, c, f, g, h \gamma_{\chi} x, y, 1\right)^{2}
$$

En prenant $h=0$, on a

$$
\mathfrak{S}=a b, \quad \mathfrak{F}=-a f, \quad(\mathfrak{S}=-b g,
$$

et les formules deviennent

$$
\left.\begin{array}{l}
\left(1+q^{2} a b\right) x^{\prime}=\left(1-q^{2} a b\right) x+2 q b y+2 q(f-q b g) \\
\left(1+q^{2} a b\right) y^{\prime}=\quad-2 q a x+\left(1-q^{2} a b\right) y-2 q(g+q a f)
\end{array}\right\}
$$

valeurs qui satisfont identiquement à l'équation

$$
\left(a x^{\prime 2}+2 g x^{\prime}+l\right)+\left(b y^{\prime 2}+2 f y^{\prime}+m\right)=\left(a x^{2}+2 g x+l\right)+\left(b y^{2}+2 f y+m\right)
$$

[où pour $c$ j'ai mis $l+m$ ] et en y écrivant

$$
\frac{1-q^{2} a b}{1+q^{2} a b}=s=\sqrt{1-a b r^{2}}
$$

on obtient des formules qui correspondent précisément aux équations données par Euler pour $x, y$ en termes de $a, b$.

Londres, 10 Mars, 1857. 